\title{
Antibiotic prophylaxis in beta-lactam allergic patients undergoing cesarean and vaginal delivery: An opportunity for stewardship
}

\author{
Daisuke Furukawa MD, MSHPM ${ }^{1,2}$ (1), Nicole Douglas MPH, CPHQ ${ }^{3}$, Jessica Hsu MD, MBA ${ }^{2,4}$, Matthew Davis PharmD ${ }^{1,5}$, \\ Christine Pham PharmD ${ }^{1,5}$, Meganne Kanatani PharmD ${ }^{1,5}$ and Tara Vijayan MD, MPH $^{1,2}$ \\ ${ }^{1}$ Division of Infectious Diseases, University of California, Los Angeles, California, ${ }^{2}$ David Geffen School of Medicine, University of California, Los Angeles, \\ California, ${ }^{3}$ Quality Management Services, UCLA Health, Los Angeles, California, ${ }^{4}$ Department of Obstetrics and Gynecology, University of California, Los Angeles, \\ California and ${ }^{5}$ Department of Pharmaceutical Services, University of California, Los Angeles, California
}

To the Editor - It is now known that the R1 group side chain is the predominant allergenic determinant in patients with a $\beta$-lactam allergy and that patients with a confirmed IgE-mediated $\beta$-lactam allergy can safely receive other $\beta$-lactams that do not share similar R1 side-chain structures. ${ }^{1,2}$ Cefazolin in particular has a distinct R1 structure and is thought to be safe to administer even in patients with a history of anaphylaxis to other $\beta$-lactams. ${ }^{1,2}$ Based on this knowledge, multiple studies have successfully increased rates of cefazolin use for perioperative prophylaxis in patients with a $\beta$-lactam allergy. 3,4

No studies have specifically applied this knowledge to obstetrics and gynecology. Cefazolin is the antibiotic of choice for cesarean section prophylaxis and is also the recommended alternative to penicillin for patients with a "low risk" penicillin allergy who require peripartum group B Streptococcus (GBS) prophylaxis., We conducted a retrospective study to identify opportunities for stewardship in $\beta$-lactam allergic patients undergoing cesarean and vaginal delivery based on our knowledge of $\beta$-lactam cross reactivity.

\section{Methods}

Medical records of patients who delivered at 1 of 2 academic medical centers in Los Angeles from January 1, 2017, to November 30, 2020, were retrospectively reviewed. Patient allergy, type of allergic reaction, delivery type (vaginal vs cesarean), GBS screening status, and antibiotics administered during the hospitalization were electronically abstracted. A descriptive analysis of patients' allergies and prophylactic antibiotics administered were performed on 2 specific subgroups: (1) patients with a $\beta$-lactam allergy label who underwent cesarean section and (2) patients with a $\beta$-lactam allergy label with a positive GBS screen who underwent vaginal delivery. Patients with suspected active peri-partum infection were excluded. This study was conducted as a quality improvement initiative.

\section{Results}

In total, 8,749 patients underwent cesarean or vaginal delivery. Of these, 827 patients $(9.5 \%)$ had a $\beta$-lactam allergy label. A list of antibiotics to which patients were allergic can be found in Supplementary Table 1 (online). Of these 827 patients, 360 met our prespecified subgroups: 260 patients who underwent cesarean delivery and 100 patients who were GBS positive and delivered

\footnotetext{
Author for correspondence: Daisuke Furukawa, E-mail: daiskfurukawa@gmail.com Cite this article: Furukawa D, et al. (2022). Antibiotic prophylaxis in beta-lactam allergic patients undergoing cesarean and vaginal delivery: An opportunity for stewardship. Infection Control \& Hospital Epidemiology, 43: 1514-1515, https://doi.org/ $10.1017 /$ ice. 2021.315
}

vaginally. Of the 260 patients that underwent cesarean delivery, $165(63.5 \%)$ received a non- $\beta$-lactam alternative (vancomycin or clindamycin) for perioperative prophylaxis. Of the 100 patients who underwent vaginal delivery, 64 (64.0\%) received a non- $\beta$-lactam alternative for GBS prophylaxis.

The documented allergic reactions are listed in Table 1 for patients who received a non- $\beta$-lactam alternative. Rash $(n=60$, $38.0 \%)$ and hives $(n=27,43.5 \%)$ were the most common documented reactions for patients that underwent cesarean and vaginal delivery, respectively. Only 5 patients $(2.3 \%$ of total) reported an allergic reaction whereby cefazolin would not have been recommended, and conversely, 215 (97.7\%) patients reported reactions whereby cefazolin likely could have been safely administered.

\section{Discussion}

History of $\beta$-lactam allergy was common in our cohort, seen in 9.5\% of patients that underwent Cesarean or vaginal delivery. Additionally, we found that many patients in our cohort who received a non- $\beta$-lactam alternative had documented reactions that were unknown or suggestive of an intolerance rather than a true allergy. These findings are consistent with studies in the literature, which have demonstrated that most patients with an allergy label do not actually have a true allergy. ${ }^{7}$ Our study highlights an important opportunity for antibiotic allergy de-labeling initiatives in the obstetrics population.

Previous studies have evaluated inappropriate or guideline discordant antibiotic use for penicillin allergic patients in the obstetrics population. ${ }^{8,9}$ However, to our knowledge, our study is the first to evaluate appropriateness of antibiotics based on side-chain cross reactivity, and we found that most patients who received a non- $\beta$-lactam prophylaxis likely could have safely received cefazolin. Given the inferior outcomes and greater side-effect profile of non- $\beta$-lactam alternatives, ${ }^{10}$ it is imperative that efforts be made to maximize $\beta$-lactam utilization in patients with a $\beta$-lactam allergy label.

Based on these data, we devised an algorithm (Supplementary Fig. $1 \mathrm{a}$ and $\mathrm{b}$ online) to help providers make appropriate antibiotic selections for patients with a $\beta$-lactam allergy label at our institution. Notably, this approach, based on side-chain cross reactivity, is not reflected in the recent American College of Obstetrics and Gynecology (ACOG) guidelines., ${ }^{5,6}$ For example, in the ACOG guidelines, cefazolin is not recommended for GBS prophylaxis in patients with a history of severe IgE-mediated penicillin allergy, whereas cefazolin is considered safe in our algorithm. Despite this deviation from guidelines, feedback from obstetrics and gynecology colleagues at our institution have been favorable with no 
Table 1. Recorded Allergic Reactions to $\beta$-Lactams for Patients Who Received a Non- $\beta$-Lactam Alternative

\begin{tabular}{lrc}
\hline Reaction & $\begin{array}{c}\text { Cesarean Delivery } \\
\left(\mathrm{n}=158^{\mathrm{a}, \mathrm{b}}\right)\end{array}$ & $\begin{array}{c}\text { GBS }+ \text { Vaginal Delivery } \\
\left(\mathrm{n}=62^{\mathrm{a}, \mathrm{b}}\right)\end{array}$ \\
\hline Cefazolin recommended & & \\
\hline IgE mediated, no. (\%) & $12(7.6)$ & $3(4.8)$ \\
\hline Anaphylaxis & $1(0.6)$ & $0(0.0)$ \\
\hline Angioedema & $60(38.0)$ & $20(32.3)$ \\
\hline Rash & $55(34.8)$ & $27(43.5)$ \\
\hline Hives & $8(5.1)$ & $2(3.2)$ \\
\hline Throat swelling & $7(4.4)$ & $1(1.6)$ \\
\hline Shortness of breath & $1(0.6)$ & $1(1.6)$ \\
\hline Wheezing & & $8(12.9)$ \\
\hline $\begin{array}{l}\text { Intolerance or unknown, } \\
\text { no. (\%) }\end{array}$ & $11(7.0)$ & $1(1.6)$ \\
\hline Itching & $1(0.6)$ & $0(0.0)$ \\
\hline Diarrhea & $2(1.3)$ & $2(3.2)$ \\
\hline Dizziness & $6(3.8)$ & $10(17.7)$ \\
\hline Nausea & $32(20.3)$ & \\
\hline Unknown & $1(0.6)$ & \\
\hline Cefazolin not recommended & \\
\hline $\begin{array}{l}\text { Severe non-IgE reaction, } \\
\text { no. (\%) }\end{array}$ & & \\
\hline Fever & & \\
\hline Hemolysis & & \\
\hline
\end{tabular}

Note. GBS, group B Streptococcus.

aPatients with cefazolin or "cephalosporin" allergy were excluded from this table.

bSum of number of patients experiencing each reaction is greater than total number of patients as one patient can endorse $>1$ reaction.

${ }^{\mathrm{C} C}$ efazolin was not recommended only when patients reported an allergic reaction suggestive of a severe non-IgE mediated reaction such as Stevens-Johnson syndrome, DRESS syndrome, hemolytic anemia, drug fever, serum sickness, etc.

reported safety concerns to date. We recommend that other institutions take similar actions to engage obstetrics and gynecology colleagues to improve prophylactic antibiotic selection in patients with a $\beta$-lactam allergy.

Our study has limitations. First, the retrospective study design limited our analysis of allergy history to what was recorded in the medical records. Thus, we may have overestimated the number of patients with an unknown reaction. However, even if we had conservatively concluded that patients with unknown allergy reaction were not suitable for cefazolin use, cefazolin would have still been acceptable in most patients and our conclusions would have remained unchanged. Second, the study was conducted in a single institution and generalizability may be limited.

In conclusion, we demonstrated that $\beta$-lactam allergy is common in pregnant patients, but most patients who received a non- $\beta$-lactam alternative for cesarean or GBS prophylaxis likely could have safely received cefazolin based on our knowledge of $\beta$-lactam cross reactivity. $\beta$-lactam allergy in pregnancy is a prime opportunity for future stewardship interventions.

Supplementary material. To view supplementary material for this article, please visit https://doi.org/10.1017/ice.2021.315

\section{Acknowledgments.}

Financial support. D.F. was funded by Health Resources and Services Administration Ruth L. Kirschstein-National Research Service (award no. T32HPI19001).

Conflicts of interest. All authors report no conflicts of interest relevant to this article.

\section{References}

1. Zagursky RJ, Pichichero ME. Cross-reactivity in beta-lactam allergy. J Allergy Clin Immunol Pract 2018;6:72-81.

2. Vorobeichik L, Weber EA, Tarshis J. Misconceptions surrounding penicillin allergy: implications for anesthesiologists. Anesth Analg 2018;127:642-649.

3. Lam PW, Tarighi P, Elligsen M, et al. Impact of Allergy Clarification for Cefazolin Evidence-based Prescribing Tool (ACCEPT) on receipt of preferred perioperative prophylaxis: an interrupted time series study. Clin Infect Dis 2020;71:2955-2957.

4. Collins CD, Scheidel C, Anam K, et al. Impact of an antibiotic side chainbased cross-reactivity chart combined with enhanced allergy assessment processes for surgical prophylaxis antimicrobials in patients with beta-lactam allergies. Clin Infect Dis 2021;72:1404-1412.

5. Prevention of group B streptococcal early-onset disease in newborns: ACOG committee opinion, number 797. Obstet Gynecol 2020;135:e51-e72.

6. Committee on Practice Bulletins-Obstetrics. ACOG practice bulletin no. 199: use of prophylactic antibiotics in labor and delivery. Obstet Gynecol 2018;132:e103-e119.

7. Sacco KA, Bates A, Brigham TJ, Imam JS, Burton MC. Clinical outcomes following inpatient penicillin allergy testing: a systematic review and meta-analysis. Allergy 2017;72:1288-1296.

8. Paccione KA, Wiesenfeld. Guideline adherence for intrapartum group B streptococci prophylaxis in penicillin-allergic patients. Infect Dis Obstet Gynecol 2013. doi: 10.1155/2013/917304.

9. Desravines N, Venkatesh KK, Hopkins A, et al. Intrapartum group B Streptococcus antibiotic prophylaxis in penicillin allergic pregnant women. AJP Rep 2019;9:e238-e243.

10. KG Blumenthal, Ryan EE, Li Y, Lee H, Kuhlen JL, Shenoy ES. The impact of a reported penicillin allergy on surgical site infection risk. Clin Infect Dis 2018;66:329-336. 